\title{
Time-Dependent Queue-Size Distribution in a Finite-Buffer Model with Server Setup Times
}

\author{
Wojciech M. Kempa \\ Silesian University of Technology, \\ Institute of Mathematics, \\ ul. Kaszubska 23, 44-100 Gliwice, Poland, \\ Email: wojciech.kempa@polsl.pl
}

\author{
Dariusz Kurzyk \\ Silesian University of Technology, \\ Institute of Mathematics, \\ ul. Kaszubska 23, 44-100 Gliwice, Poland, \\ Institute of Theoretical and Applied Informatics, \\ Polish Academy of Sciences, \\ ul. Bałtycka 5, 44-100 Gliwice, Poland, \\ Email: dariusz.kurzyk@polsl.pl
}

\begin{abstract}
Transient queue-size distribution in a finite-buffer system with Poisson arrivals and generally distributed processing times is investigated. In the evolution of the system the server needs randomly distributed setup times preceding the service initialization in each new busy period. Applying the paradigm of embedded Markov chain and the formula of total probability, a Volterra-type system of integral equations for the transient queue-size distribution, conditioned by the number of packets being accumulated in the buffer before the opening of the system, is built. The solution of the corresponding system written for Laplace transforms is obtained algebraically in the compact explicit form. Numerical examples are attached as well.
\end{abstract}

\section{INTRODUCTION}

$\mathbf{F}$ INITE-BUFFER queues are widely used in modelling real-life processing systems, in which the phenomena like buffering of waiting packets (jobs, customers, calls, etc.), delays of different nature, and packet losses caused by buffer overflows or temporary suspensions of the service process occur. In particular, e.g. the incoming/outcoming stream of packets in a node of computer network (like, e.g. IP router in the Internet) can be efficiently modelled by using one of finite queueing systems. One of the most important challenges of wireless communication (e.g., based on Wi-Fi IEEE.802.11 standard or wireless sensor networks), is the problem of energy saving. In practice, a mechanism for temporary switching off the radio transmitting/receiving, e.g. at a time at which the stream of packets directed to this node becomes less intensive or when the accumulating buffer is empty, is being implemented. After such a time of unavailability, to start the processing normally, a node needs some period of time (called a setup time, usually random) to achieve full readiness to work. During the setup time the service process is blocked and the arriving packets accumulate in the buffer queue. Such a mechanism can be observed, e.g. in manufacturing systems or in the GSM standard transmission in which the node is being switched on just before sending the identification frame by a BS (=Base Station).

In [10] a steady-state threshold strategy for jobs behavior in a model with setup times is obtained. One can find the study of a Markovian system with server setups preceding the first processing in each busy period in [6]. Different results on queueing models with setup times applied in the analysis of WSNs' operation are derived, e.g. in [11] and [12]. In [12] the model of a sleep/wakeup protocol in the IEEE 802.15.4 can be found (see also [7], [9] and [13] for some other results concerning the energy saving problem in WSNs). The IMS session re-setup delay in WiMAX/LTE heterogeneous networks is modelled by an appropriate queueing system in [1]. The $M / G / 1$-type queue with vacation policy and server setup times is used in [8] for modelling the BS sleeping mode in cellular networks. In [2] a model of data center with servers leaving their idle periods "via" setup times is investigated.

The non-stationary study of the queue-size distribution in the $M / G / 1$-type queueing system with random batch arrivals, $N$-policy and server setup times can be found in [3] (see also [4], where departure process is investigated).

In the paper we investigate the finite-buffer $M / G / 1$-type queueing system in which the first service beginning each new busy period is preceded by a generally-distributed setup time, during which the processing is still blocked and the server acquires full readiness for the service process. Applying the approach based on the idea of embedded Markov chain and the total probability law, a system of integral equations for the transient queue-size distribution, conditioned by the initial buffer state, is built. The solution of the corresponding system written for Laplace transforms is obtained by using the linear algebraic approach and given in a closed form, utilizing a certain functional sequence defined recursively.

\section{Model Description And Auxiliary Results}

In the article we deal with a single-server finite-buffer queueing model, in which packets occur according to a Poisson process with intensity $\lambda$ and are being processed individually with a general-type $\mathrm{CDF}$ (=cumulative distribution function) $F(\cdot)$ of the service time, according to the FIFO discipline. The total number of packets present in the system simultaneously is bounded by a non-random value $K$, i.e. we have $K-1$ places in the buffer queue and one place "in processing". As it is usually assumed, packets arriving during the buffer overflow period, i.e. when the server is busy with processing and the buffer is saturated, are being lost. In general, it is allowed for 
the buffer to contain a number of packets being waiting before the opening of the system at time $t=0$. Each busy period, which starts together with the first arrival after the idle time, is preceded by a random setup time with a $\operatorname{CDF} G(\cdot)$ of a general type. The setup time is a period during which the processing is blocked and the server "acquires" full readiness for processing. It is assumed that the system being empty before the start also initializes a setup time at the first arrival epoch. We assume, moreover, that all interarrival, processing and setup times in the evolution of the system are independent.

Let us denote by $X(t)$ the number of packets present in the system at time $t$, including a packet being processed at this time (if any). Define the transient distribution function of $X(t)$, conditioned by the initial level of buffer saturation, in the following way:

$$
Q_{n}(t, m) \stackrel{\text { def }}{=} \mathbf{P}\{X(t)=m \mid X(0)=n\} d t,
$$

where $t>0,0 \leq n \leq K$ and $m \geq 0$. We are interested in finding the closed-form representation for the LT (=Laplace transform) of $Q_{n}(t, m)$, i.e. for the functional

$$
\widetilde{q}_{n}(s, m) \stackrel{\text { def }}{=} \int_{0}^{\infty} e^{-s t} Q_{n}(t, m) d t, \quad \operatorname{Re}(s)>0 .
$$

In the analytical approach we use the following result from linear algebra which can be found in [5]:

Lemma 1. Let $\left(\alpha_{k}\right), k \geq 0$, and $\left(\phi_{k}\right), k \geq 1$, with the assumption $\alpha_{0} \neq 0$, be two given number sequences. Each solution of the following system of linear equations:

$$
\sum_{k=-1}^{n} \alpha_{k+1} x_{n-k}-x_{n}=\phi_{n}, \quad n \geq 0,
$$

can be written in the form

$$
x_{n}=C R_{n+1}+\sum_{k=0}^{n} R_{n-k} \phi_{k}, \quad n \geq 0,
$$

where $C$ is a constant independent on $n$, and $\left(R_{k}\right)$ is the sequence (which is called in [5] a potential) associated with the sequence $\left(\alpha_{k}\right)$ as in the following relationships:

$$
\begin{aligned}
& R_{0}=0, \quad R_{1}=\alpha_{0}^{-1} \\
& R_{k+1}=\alpha_{0}^{-1}\left(R_{k}-\sum_{i=0}^{k} \alpha_{i+1} R_{k-i}\right), \quad k \geq 1 .
\end{aligned}
$$

We end this section with stating some additional notations. We use the abbreviation $\bar{G}(x) \stackrel{\text { def }}{=} 1-G(x)$ and the nomenclature $I\{\mathbb{A}\}$ for the indicator of the random event $\mathbb{A}$. Besides, let us denote by $f(\cdot)$ and $g(\cdot)$ the Laplace-Stieltjes transforms of CDFs $F(\cdot)$ and $G(\cdot)$, respecively.

\section{System OF EQUATIONS FOR CONDITIONAL QueUE-SizE Distribution}

Let us start with the case of the system being empty at time $t=0$. Thus, the evolution of the system begins with an idle period during which the service station "waits" for packets. Simultaneously with the arrival occurrence a setup time begins.
Let us note that we can distinguish three mutually excluding situations (random events):

(1) the first packet arrives before time $t$ and the setup time also ends before $t$ (we denote this event by $A_{1}(t)$ );

(2) the first packet enters before $t$ but the setup time completes after $t\left(A_{2}(t)\right)$;

(3) the first packet arrives after time $t\left(A_{3}(t)\right)$.

Introduce the following notation:

$$
Q_{0}^{(i)}(t, m)=\mathbf{P}\left\{(X(t)=m) \cap A_{i}(t) \mid X(0)=0\right\},
$$

where $t>0, m \geq 0$ and $i=1,2,3$. It is obvious that the formula of total probability leads to the following relationship:

$$
Q_{0}(t, m)=\mathbf{P}\{X(t)=m \mid X(0)=0\}=\sum_{i=1}^{3} Q_{0}^{(i)}(t, m)
$$

and, moreover,

$$
\widetilde{q}_{0}(s, z)=\sum_{i=1}^{3} \int_{0}^{\infty} e^{-s t} Q_{0}^{(i)}(t, m) d t .
$$

Considering the random event $A_{1}(t)$, we get the following equation:

$$
\begin{aligned}
& Q_{0}^{(1)}(t, m)=\int_{x=0}^{t} \lambda e^{-\lambda x} d x \\
& \times \int_{y=0}^{t-x}\left[\sum_{i=0}^{K-2} \frac{(\lambda y)^{i}}{i !} e^{-\lambda y} Q_{i+1}(t-x-y, m)\right. \\
& \left.+Q_{K}(t-x-y, m) \sum_{i=K-1}^{\infty} \frac{(\lambda y)^{i}}{i !} e^{-\lambda y}\right] d G(y) .
\end{aligned}
$$

Let us note that the first summand on the right side of (9) corresponds to the situation in which there is at least one free place in the buffer at the completion epoch of the setup time. The second summand presents the case in that the buffer becomes saturated during the setup time.

Similarly, for $A_{2}(t)$, we obtain

$$
\begin{aligned}
& Q_{0}^{(2)}(t, m)=\int_{0}^{t} \lambda e^{-\lambda x} \bar{G}(t-x) \\
& \times\left\{I\{1 \leq m \leq K-1\} \frac{[\lambda(t-x)]^{m-1}}{(m-1) !} e^{-\lambda(t-x)}\right. \\
& \left.+I\{m=K\} \sum_{i=K-1}^{\infty} \frac{[\lambda(t-x)]^{i}}{i !} e^{-\lambda(t-x)}\right\} d x .
\end{aligned}
$$

Let us comment (10) briefly. The first summand under the integral on the right side of (10) relates to the case when the number of packets, measured at time $t$, is less than the maximal value $K$. The second one concerns the situation $m=$ $K$, so during the time $(t-x)$ (the time between the first arrival epoch and $t$ ) at least $K-1$ packets must occur. However, $K-1$ packets only are physically buffered. The remaining ones will be lost due to the buffer saturation.

Finally we have, obviously,

$$
Q_{0}^{(3)}(t, m)=I\{m=0\} e^{-\lambda t} .
$$


From (9)-(11), referring to (7), we get

$$
\begin{aligned}
& Q_{0}(t, m)=\int_{x=0}^{t} \lambda e^{-\lambda x} d x \\
& \times \int_{y=0}^{t-x}\left[\sum_{i=0}^{K-2} \frac{(\lambda y)^{i}}{i !} e^{-\lambda y} Q_{i+1}(t-x-y, m)\right. \\
& \left.+Q_{K}(t-x-y, m) \sum_{i=K-1}^{\infty} \frac{(\lambda y)^{i}}{i !} e^{-\lambda y}\right] d G(y) \\
& +\int_{0}^{t} \lambda e^{-\lambda x} \bar{G}(t-x)\{I\{1 \leq m \leq K-1\} \\
& \times \frac{[\lambda(t-x)]^{m-1}}{(m-1) !} e^{-\lambda(t-x)}+I\{m=K\} \sum_{i=K-1}^{\infty} \frac{[\lambda(t-x)]^{i}}{i !} \\
& \left.\times e^{-\lambda(t-x)}\right\} d x+I\{m=0\} e^{-\lambda t} .
\end{aligned}
$$

Investigate now the case of the system being non-empty at the start moment (i.e. $1 \leq n \leq K$ ). Due to the fact that successive departure epochs are Markov (renewal) moments during the operation of the $M / G / 1$-type system, then, by virtue of the continuous version of the total probability law, applied with respect to the first departure moment after $t=0$, we obtain the following system of integral equations for $1 \leq n \leq K$ :

$$
\begin{aligned}
& Q_{n}(t, m)=\int_{0}^{t}\left[\sum_{i=0}^{K-n-1} \frac{(\lambda x)^{i}}{i !} e^{-\lambda x} Q_{n+i-1}(t-x, m)\right. \\
& \left.+Q_{K-1}(t-x, m) \sum_{i=K-n}^{\infty} \frac{(\lambda x)^{i}}{i !} e^{-\lambda x}\right] d F(x) \\
& +\bar{F}(t)\left[I\{n \leq m \leq K-1\} \frac{(\lambda t)^{m-n}}{(m-n) !} e^{-\lambda t}\right. \\
& \left.+I\{m=K\} \sum_{i=K-n}^{\infty} \frac{(\lambda t)^{i}}{i !} e^{-\lambda t}\right],
\end{aligned}
$$

It is easy to note that the first summand under the integral on the right side of (13) describes the case in which the buffer does not become saturated before the first departure time $0<$ $x<t$, while the second one relates to the opposite situation. In the last summand the first service completes after $t$.

Observe that the following identity is true (compare (9)):

$$
\begin{aligned}
& \int_{t=0}^{\infty} e^{-s t} d t \int_{x=0}^{t} \lambda e^{-\lambda x} d x \int_{y=0}^{t-x} \frac{(\lambda y)^{i}}{i !} e^{-\lambda y} \\
& \times Q_{j}(t-x-y, m) d G(y)=a_{i}(s) \widetilde{q}_{j}(s, m),
\end{aligned}
$$

where

$$
a_{i}(s) \stackrel{\text { def }}{=} \frac{\lambda}{\lambda+s} \int_{0}^{\infty} \frac{(\lambda y)^{i}}{i !} e^{-(\lambda+s) y} d G(y) .
$$

Similarly, defining (see (10))

$$
\bar{a}_{i}(s) \stackrel{\text { def }}{=} \frac{\lambda}{\lambda+s} \int_{0}^{\infty} \frac{(\lambda u)^{i}}{i !} e^{-(\lambda+s) u} \bar{G}(u) d u,
$$

and taking into consideration (14)-(15), we rewrite the equation (12) in the following form:

$$
\begin{aligned}
& \widetilde{q}_{0}(s, m)=\sum_{i=0}^{K-2} a_{i}(s) \widetilde{q}_{i+1}(s, m) \\
& +\widetilde{q}_{K}(s, m) \sum_{i=K-1}^{\infty} a_{i}(s)+\beta(s, m)
\end{aligned}
$$

where

$$
\begin{aligned}
& \beta(s, m) \stackrel{\text { def }}{=} I\{1 \leq m \leq K-1\} \bar{a}_{m-1}(s) \\
& +I\{m=K\} \sum_{i=K-1}^{\infty} \bar{a}_{i}(s)+I\{m=0\} \frac{1}{\lambda+s} .
\end{aligned}
$$

Similarly, denoting

$$
\begin{aligned}
& \alpha_{i}(s) \stackrel{\text { def }}{=} \int_{0}^{\infty} e^{-(\lambda+s) x} \frac{(\lambda x)^{i}}{i !} d F(x) \\
& \gamma_{n}(s, m) \stackrel{\text { def }}{=} \int_{0}^{\infty} e^{-(\lambda+s) t} \bar{F}(t)[I\{n \leq m \leq K-1\} \\
& \left.\times \frac{(\lambda t)^{m-n}}{(m-n) !}+I\{m=K\} \sum_{i=K-n}^{\infty} \frac{(\lambda t)^{i}}{i !}\right] d t
\end{aligned}
$$

where $\operatorname{Re}(s)>0$, we transform (13) as follows:

$$
\begin{aligned}
& \widetilde{q}_{n}(s, m)=\sum_{i=0}^{K-n-1} \alpha_{i}(s) \widetilde{q}_{n+i-1}(s, m) \\
& +\widetilde{q}_{K-1}(s, m) \sum_{i=K-n}^{\infty} \alpha_{i}(s)+\gamma_{n}(s, m) .
\end{aligned}
$$

Let us apply to (17) and (21) the following substitution:

$$
\widetilde{u}_{n}(s, m) \stackrel{\text { def }}{=} \widetilde{q}_{K-n}(s, m), \quad 0 \leq n \leq K .
$$

After this operation, we get from (21) the following system:

$$
\sum_{i=-1}^{n} \alpha_{i+1}(s) \widetilde{u}_{n-i}(s, m)-\widetilde{u}_{n}(s, m)=\phi_{n}(s, m)
$$

where $0 \leq n \leq K-1$, and

$$
\begin{aligned}
& \phi_{n}(s, m) \stackrel{\text { def }}{=} \alpha_{n+1}(s) \widetilde{u}_{0}(s, m) \\
& -\widetilde{u}_{1}(s, m) \sum_{i=n+1}^{\infty} \alpha_{i}(s)-\gamma_{K-n}(s, m) .
\end{aligned}
$$

In the same manner, inserting (22) into (17), we obtain

$$
\begin{aligned}
& \widetilde{u}_{K}(s, m)=\sum_{i=0}^{K-2} a_{i}(s) \widetilde{u}_{K-i-1}(s, m) \\
& +\widetilde{u}_{0}(s, m) \sum_{i=K-1}^{\infty} a_{i}(s)+\beta(s, m) .
\end{aligned}
$$




\section{MAIN RESUltS FOR TRANSFORMS}

Let us note that (23) has the same form as (3), but with coefficients $\alpha_{i}(\cdot)$ and $\phi_{i}(\cdot, \cdot), i \geq 0$, depending on $s$ and $(s, m)$, respectively. So, there is possible to solve (23) by utilizing the result (4). Moreover, due to the fact that the number of equations in (23) is finite comparing to (3), the representation for $C=C(s, m)$ can be found explicitly, considering the last equation (25) as a special-type boundary condition. According to (4), we obtain for $n \geq 0$

$$
\widetilde{u}_{n}(s, m)=C(s, m) R_{n+1}(s)+\sum_{i=0}^{n} R_{n-i}(s) \phi_{i}(s, m),
$$

where the sequence $\left(R_{k}(s)\right)$ is defined in (5) (with $\alpha_{k}(s)$ instead of $\alpha_{k}$ ). Substituting $n=0$ into (26), we get

$$
\widetilde{u}_{0}(s, m)=C(s, m) R_{1}(s) .
$$

Taking $n=1$ in (26) and referring to (24) and (27), we obtain

$$
\begin{aligned}
& \widetilde{u}_{1}(s, m)=C(s, m) R_{2}(s)+R_{1}(s)\left(\alpha_{1}(s) R_{1}(s) C(s, m)\right. \\
& \left.-\widetilde{u}_{1}(s, m) \sum_{i=1}^{\infty} \alpha_{i}(s)-\gamma_{K}(s, m)\right)
\end{aligned}
$$

that leads to

$$
\begin{aligned}
& \widetilde{u}_{1}(s, m)=\eta(s)\left[C(s, m)\left(R_{2}(s)+\alpha_{1}(s) R_{1}^{2}(s)\right)\right. \\
& \left.-R_{1}(s) \gamma_{K}(s, m)\right],
\end{aligned}
$$

where $\eta(s) \stackrel{\text { def }}{=} \frac{f(\lambda+s)}{f(s)}$. Since using (27) and (29) the functionals $\phi_{i}(s, m), i \geq 0$, can be found, if only the formula for $C(s, m)$ is known, the key problem is in finding the explicit representation for $C(s, m)$. By using the representations (24) and (26), we can present (25) in the following form:

$$
\widetilde{u}_{K}(s, m)=\Delta_{1}(s) C(s, m)+\kappa_{1}(s, m),
$$

where we denote

$$
\begin{aligned}
& \Delta_{1}(s) \stackrel{\text { def }}{=} \sum_{i=1}^{K-1} a_{K-i-1}(s)\left[R_{i+1}(s)+\sum_{j=0}^{i} R_{i-j}(s)\right. \\
& \times\left(R_{1}(s) \alpha_{j+1}(s)-\eta(s)\left(R_{2}(s)+\alpha_{1}(s) R_{1}^{2}(s)\right) \sum_{r=j+1}^{\infty} \alpha_{r}(s)\right] \\
& +R_{1}(s) \sum_{i=K-1}^{\infty} a_{i}(s)
\end{aligned}
$$

and

$$
\begin{aligned}
& \kappa_{1}(s, m) \stackrel{\text { def }}{=} \sum_{i=1}^{K-1} a_{K-i-1} \sum_{j=0}^{i} R_{i-j}(s)\left[R_{1}(s) \gamma_{K}(s, m) \eta(s)\right. \\
& \left.\sum_{r=j+1}^{\infty} \alpha_{r}(s)-\gamma_{K-j}(s, m)\right]+\beta(s, m)
\end{aligned}
$$

Similarly, let us take $n=K$ in (26) and apply the formulae (24), (27) and (29). In consequence we obtain

$$
\widetilde{u}_{K}(s, m)=\Delta_{2}(s) C(s, m)+\kappa_{2}(s, m),
$$

where

$$
\begin{aligned}
& \Delta_{2}(s) \stackrel{\text { def }}{=} R_{K+1}(s)+\sum_{i=0}^{K} R_{K-i}(s)\left[\alpha_{i+1}(s) R_{1}(s)\right. \\
& \left.-\eta(s)\left(R_{2}(s)+\alpha_{1}(s) R_{1}^{2}(s)\right) \sum_{j=i+1}^{\infty} \alpha_{j}(s)\right]
\end{aligned}
$$

and

$$
\begin{aligned}
& \kappa_{2}(s, m) \stackrel{\text { def }}{=} \sum_{i=0}^{K} R_{K-i}(s)\left(\eta(s) R_{1}(s) \gamma_{K}(s, m)\right. \\
& \left.\times \sum_{j=i+1}^{\infty} \alpha_{j}(s)-\gamma_{K-i}(s, m)\right) .
\end{aligned}
$$

Comparing the right sides of (30) and (33), we express $C(s, m)$ as follows:

$$
C(s, m)=\frac{\kappa_{2}(s, m)-\kappa_{1}(s, m)}{\Delta_{1}(s)-\Delta_{2}(s)} .
$$

Now the formulae (24), (26), (27), (29) and (36) lead to the following main theorem:

Theorem 1. The representation for the $L T$ of the conditional transient queue-size distribution in the $M / G / 1 / K$-type model with generally distributed setup times is following:

$$
\begin{aligned}
& \widetilde{q}_{n}(s, m)=\int_{0}^{\infty} e^{-s t} \mathbf{P}\{X(t)=m \mid X(0)=n\} d t \\
& =\frac{\kappa_{2}(s, m)-\kappa_{1}(s, m)}{\Delta_{1}(s)-\Delta_{2}(s)}\left\{R_{K-n+1}(s)+\sum_{i=0}^{K-n} R_{K-n-i}(s)\right. \\
& \times\left[\alpha_{i+1}(s) R_{1}(s)-\eta(s)\left(R_{2}(s)+\alpha_{1}(s) R_{1}^{2}(s)\right)\right. \\
& \left.\left.\times \sum_{j=i+1}^{\infty} \alpha_{j}(s)\right]\right\}+\sum_{i=0}^{K-n} R_{K-n-i}(s)\left(\eta(s) R_{1}(s) \gamma_{K}(s, m)\right. \\
& \left.\times \sum_{j=i+1}^{\infty} \alpha_{j}(s)-\gamma_{K-i}(s, m)\right),
\end{aligned}
$$

where the formulae for $\alpha_{i}(s), \gamma_{i}(s, m), R_{i}(s), \Delta_{1}(s)$, $\kappa_{1}(s, m), \Delta_{2}(s)$ and $\kappa_{2}(s, m)$ are given in (19), (20), (5), (31), (32), (34) and (35), respectively.

Remark IV.1. Let us note that from the formula (37) the stationary queue-size distribution $\pi_{m}, m=0, \ldots, K$, can be found by using the Tauberian theorem, namely for any $n \in\{0, \ldots, K\}$

$$
\pi_{m}=\lim _{t \rightarrow \infty} \mathbf{P}\{X(t)=m\}=\lim _{s \downarrow 0} s \cdot \widetilde{q}_{n}(s, m) .
$$

\section{NUMERICAL EXAMPLES}

Let us take into consideration a node of the wireless network with buffer of size 6 packets, with the arrival stream of packets of average sizes $200 \mathrm{~B}$, entering with intensity 600 $\mathrm{Kb} / \mathrm{s}$. Adjusting the Poisson arrival process, we have the rate $\lambda=375$ packets per second. Assume that packets are being transmitted with speed $720 \mathrm{~Kb} / \mathrm{s}$, where the processing times have 2-Erlang distribution, that gives the intensity 450 packets 
per second and the value $\mu=900$ of the parameter of this distribution. Hence, the load of the node is at the high level $(\rho=0.833)$. Moreover, we consider the mechanism of exponentially distributed setup with three different means: 0 (without setup - "pure" system), 5 and 50 [ms].

The evolution of the transient probabilities $\mathbf{P}\{X(t)=$ $m \mid X(0)=0\}$ for $m=1,3,5$ are presented in Fig. 1. Evidently, due to different durations of setup times, the transient evolutions and stationary values of the proper probabilities are different in cases (a)-(c). As one can observe, a short setup time with mean $5[\mathrm{~ms}]$ allows the system to stabilize faster than in the absence of such a period (compare cases (a) and (b)). From the other side, if the setup time is relatively long in comparison to the arrival/service rates (case (c)), the time of stabilization elongates in comparison to the system without setup time. It is worth mentioning that all analytical results are confirmed by process-based discrete-event simulations (DES).

\section{SUMMARY}

In the article a finite-buffer queueing model with Poisson arrivals and generally distributed processing times and server setup times is considered. The closed-form representation for the LT of the transient queue-size distribution conditioned by the initial buffer state is found, from which the stationary distribution can be obtained directly by using the Tauberian theorem. Numerical examples are attached as well.

\section{REFERENCES}

[1] E. P. Edward, "A Novel Seamless Handover Scheme for WiMAX/LTE Heterogeneous Networks," Arab. J. Sci. Eng., vol. 41, iss. 3, 2016, pp. 1129-1143.

[2] J. N. Hu and P. D. Tuan, "Power Consumption Analysis for data Centers with Independent Setup Times and Threshold Controls," AIP Conference Proceedings, vol. 1648, 2015.

[3] W. M. Kempa, "On Transient Queue-Size Distribution in the Batch Arrival System with the $N$-policy and Setup Times," Math. Commun., vol. 17, iss. 1, 2012, 285-302.

[4] W. M. Kempa and D. Kurzyk, "Transient Departure Process in $M / G / 1 / K$-type Queue with Threshold Server's Waking Up," Proc. of the 23rd International Conference on Software, Telecommunications and Computer Networks (SoftCOM 2015),Split - Bol (Island of Brac), Croatia, 2015, pp. 32-36, DOI: 10.1109/SOFTCOM.2015.7314127.

[5] V. S. Korolyuk, Boundary-value problems for compound Poisson processes, Naukova Dumka, Kiev, 1975.

[6] Q. Ma, "Analysis of a Clearing Queueing System with Setup Times," RAIRO-Oper. Res., vol. 49, iss. 1, 2015, pp. 67-76, DOI: http://dx.doi.org/10.1051/ro/2014035.

[7] J. Miček and J. Kapitulík, "WSN Sensor Node for Protected Area Monitoring," Proc. of FedCSIS 2012, 803-807.

[8] Z. S. Niu and X. Y. Guo and S. Zhou and P. R. Kumar, "Characterizing Energy-Delay Tradeoff in Hyper-Cellular Networks With Base Station Sleeping Control," IEEE J. Sel. Area Comm., vol. 33, iss. 4, 2015, 641650, DOI: 10.1109/JSAC.2015.2393494.

[9] M. Salayma and A. Al-Dubai and I. Romdhani and M. B. Yassein, "Battery Aware Beacon Enabled IEEE802.15.4: An Adaptive and Cross-Layer Approach," Proc. of FedCSIS 2015, 1267-1272, DOI: 10.15439/2015F118.

[10] W. Sun and P. F. Guo and N. S. Tian, "Equilibrium Threshold Strategies in Observable Queueing Systems with Setup/Closedown Times," Cent. Europ. J. Oper. Re., vol. 18, iss. 3, 2010, pp. 241-268, DOI: 10.1007/s10100-009-0104-4.

[11] Q. T. Sun and S. F. Jin and C. Chen, "Energy Analysis of Sensor Nodes in WSN Based on Discrete-Time Queueing Model with a Setup," Proc. of 22nd Chinese Control and Decision Conference, Xuzhou, China, vols. 1-5, 2010, pp. 4114-4118, DOI: 10.1109/CCDC.2010.5498425.

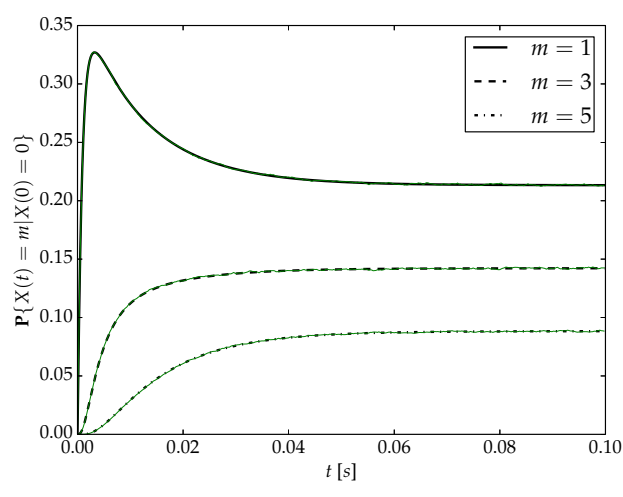

(a) no setup time

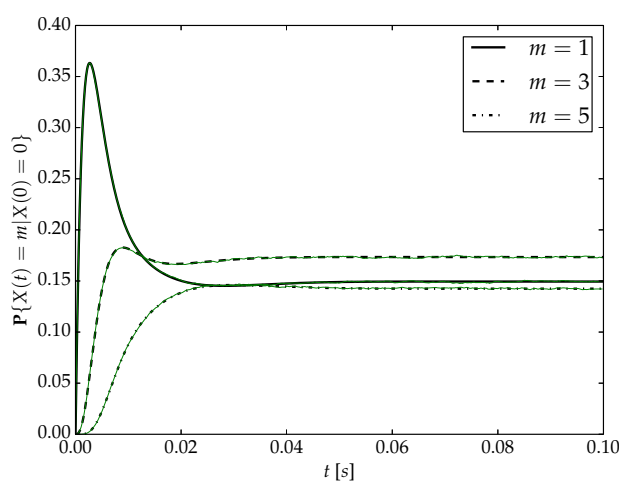

(b) setup time mean $5 \mathrm{~ms}$

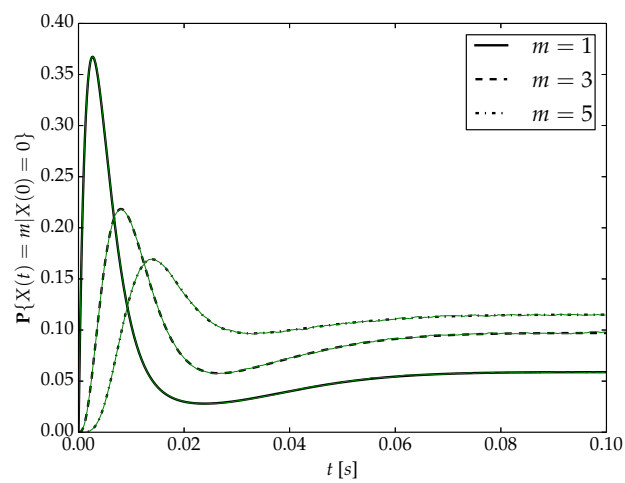

(c) setup time mean $50 \mathrm{~ms}$

Fig. 1. Subfigures (a), (b) i (c) present the probabilities $\mathbf{P}\{X(t)=$ $m \mid X(0)=0\}$ for $m=1$ (solid line), $m=3$ (dashed line) and $m=5$ (dot dashed line), for the case of no setup time (subfigure (a)), a setup time with mean 5 [ms] (subfigure (b)) and with mean 50 [ms] (subfigure (c)). Bold black lines and thin green lines correspond with analytical and DES results, respectively

[12] W. Y. Yue and Q. T. Sun and S. F. Jin, "Performance Analysis of Sensor Nodes in a WSN With Sleep/Wakeup Protocol," Lect. Notes Oper. Res., vol. 12, 2010, pp. 370-377.

[13] M. Zieliński and F. Mieyeville and D. Navarro and O. Bareille, "A Low Power Wireless Sensor Node with Vibration Sensing and Energy Harvesting Capability," Proc. of FedCSIS 2014, 1065-1071. 